\title{
Internacionalización de las universidades latinoamericanas
}

\section{Internationalization of Latin American universities}

DOI: 10.46932/sfjdv3n1-061

Received in: Jan 30st, 2021

Accepted in: Feb 1th, 2022

\author{
Carlos Rios-Campos \\ Investigador RENACYT. \\ Doctor en Gestión Universitaria. \\ Maestro en Administración. \\ Ingeniero de Sistemas.
}

Docente de la Universidad Nacional Toribio Rodríguez de Mendoza de Amazonas.

Miembro del Colegio de Ingenieros del Perú. Section Investigation Activities Chair North (IAC) - IEEE Perú.

Calle La Plata 155 - Urb: San Eduardo - Chiclayo, Perú.

E-mail: carlos.rios@untrm.edu.pe

\section{Dunia Lucía Barreiro Moreira}

Magister en Educación Superior, Investigación e Innovaciones Pedagógicas.

Docente de Educación Superior.

Trabajo en la Universidad Laica Vicente Rocafuerte de Guayaquil.

Avenida de las Américas N. 70. Guayaquil, Ecuador.

E-mail: dbarreirom@ulvr.edu.ec

\section{Liliana Isabel Flores Anchundia}

Aspirante a Doctora en Educación en la Universidad Cesar Vallejo, Piura - Perú, Ingeniera en administración financiera.

Magister en Contabilidad y Auditoría.

Egresada en Maestría de Gestión Educativa.

Docente en el Área de Contabilidad, Unidad Educativa José María Velasco Ibarra, Buena Fe, Los Ríos, Ecuador.

calle arcadio fuente y transversal sin nombre junto al cementerio general.

E-mail: lili_isaldu@hotmail.es

\section{Katerine Cecibel Muñoz Sánchez}

Ingeniera agropecuaria.

Egresada en Maestría de Gestión Educativa.

Docente en el área de Matemática, Unidad Educativa José María Velasco Ibarra, Buena Fe, Los Ríos, Ecuador.

calle arcadio fuente y transversal sin nombre junto al cementerio general.

Email: katceci4@outlookl.com.ar

\section{Oscar Efrén Anchundia Gómez}

Magister en Educación Universitaria e Investigación Educativa.

Docente de la Universidad de Guayaquil.

Universidad de Guayaquil. Facultad de Filosofia Letras y Ciencias de la Educación - Carrera Educación Básica. 
Ciudadela Ciudad del Río 2 - Mz 955 Villa 17. Parroquia Pascuales. Guayaquil, Ecuador.

E-mail: oanchundia@gmail.com

\section{Noemí Concha-Lima}

Universidad Cesar Vallejo Piura, Perú.

Magíster en Educación, con mención en docencia y gestión educativa de la Universidad Cesar Vallejo 2012 Perú.

Obtuvo el título de segunda Especialidad en monitoreo Fetal y diagnóstico por imágenes en Obstetricia de la Universidad Hermilio Valdizán Huánuco Perú 2017.

Obtuvo el título de Licenciada en obstetricia Universidad Católica Santa María Arequipa Perú. Profesión Obstetra.

Santa Margarita Mz Pe lote 21 tercera Etapa, distrito 26 de octubre, Piura.

E-mail: noemi9813@hotmail.com

Marleni del Aguila Allaín

Maestra de Educación Inicial.

Maestría.

I.E.I. 250 Nuevo Aguas Verdes.

Av. República de Chile Mz C15 Lote 1 Aguas Verdes - Zarumilla, Perú.

E-mail: mar10leni@hotmail.com

\section{Irma Rumela Aguirre Zaquinaula}

Doctora en Administración de la Educación.

Docente Ordinaria Principal de la Universidad Nacional de Jaén.

Jirón Cuzco 250 - Jaén, Perú.

E-mail: irma.aguirre@unj.edu.pe

\section{RESUMEN}

En la gestión universitaria durante el contexto actual, la internacionalización de las universidades latinoamericanas es un aspecto muy importante y urgente. En esta investigación, se planteó el objetivo general, analizar la internacionalización de las universidades latinoamericanas. Metodología, la investigación presentó un diseño cualitativo-interpretativo, de tipo documental, se han seleccionado 52 documentos, realizados en el periodo 2017 -2022, incluyendo: artículos científicos, artículos de revisión e información de sitios web de organizaciones reconocidas. Las palabras claves utilizadas en las búsquedas fueron: internacionalización, Latinoamérica, universidad. Resultados, los procesos de internacionalización en las universidades latinoamericanas, cobran mayor importancia durante la pandemia, y también, demuestran un esfuerzo sostenido de estas instituciones. La internacionalización debe priorizarse en la gestión de las universidades públicas y privadas. Conclusiones, dentro de las políticas de internacionalización de las universidades latinoamericanas, tenemos la movilidad estudiantil, investigación, educación y colaboración académica y cultural, la cooperación e integración, participar en proyectos colaborativos e intercambios académicos con universidades del extranjero, firma de convenios académicos internacionales, contribuir a una educación más inclusiva, integración de componentes internacionales e interculturales, coloquios internacionales, internacionalizar el currículo universitario, generación de circuitos regionales o redes de producción y difusión de conocimiento. Es importante y urgente, que las universidades latinoamericanas, trabajen de manera coordinada y conjunta para lograr mejores resultados en sus procesos de internacionalización.

Palabras claves: Internacionalización, Latinoamérica, Universidad. 


\begin{abstract}
In university management during the current context, the internationalization of Latin American universities is a very important and urgent aspect. In this research, the general objective was raised, to analyze the internationalization of Latin American universities. Methodology, the research presented a qualitative-interpretative design, documentary type, 52 documents have been selected, carried out in the period 2017 - 2022, including: scientific articles, review articles and information from websites of recognized organizations. The keywords used in the searches were: internationalization, Latin America, university. Results, the internationalization processes in Latin American universities become more important during the pandemic, and also demonstrate a sustained effort by these institutions. Internationalization must be prioritized in the management of public and private universities. Conclusions, within the internationalization policies of Latin American universities, we have student mobility, research, education and academic and cultural collaboration, cooperation and integration, participation in collaborative projects and academic exchanges with foreign universities, signing of international academic agreements, contribute to a more inclusive education, integration of international and intercultural components, international colloquiums, internationalize the university curriculum, generation of regional circuits or networks of production and dissemination of knowledge. It is important and urgent that Latin American universities work in a coordinated and joint manner to achieve better results in their internationalization processes.
\end{abstract}

Keywords: Internationalization, Latin America, university.

\title{
1 INTRODUCCIÓN
}

En esta investigación se planteó el objetivo general, analizar la internacionalización de las universidades latinoamericanas.

\subsection{INTERNACIONALIZACIÓN UNIVERSITARIA}

La internacionalización en las instituciones de educación superior resulta vital para el posicionamiento de las universidades a nivel global. En este sentido, el apoyo de las autoridades de la IES debe estar orientado a dar prioridad a la internacionalización y convocar a todos implicados para que participen en el diseño y la puesta en práctica de las políticas y estrategias... (Macazana et al., 2021).

Así, la internacionalización es el medio que permite a las IES tejer redes de conocimiento e investigación; apoyadas en instrumentos estratégicos, como las nuevas tecnologías de la información y la comunicación, las redes sociales, les ayuda a impulsar nuevos procesos de construcción y de difusión de conocimientos científicos, nuevos compromisos sociales en las esferas local, regional y global (Comas, 2019).

La internacionalización no debe verse como una serie de hechos aislados por medio de los cuales un número limitado de individuos tiene la experiencia de vivir fuera de su país; sino que, sus beneficios puede expandirse a más personas a través de actividades que formen parte del planeamiento educativo; 
esto es, una perspectiva internacional desde las estrategias didácticas a nivel de educación superior (López, 2018).

Abordar la internacionalización universitaria en toda su complejidad, es fundamental, para arribar a una mirada holística del fenómeno que contribuya a la planificación política y la proyección institucional (Oregioni, 2017).

Con la llegada de la enfermedad COVID-19, la internacionalización y la movilidad estudiantil se encuentran ante un future incierto, ante la urgencia de resolver otras necesidades en las instituciones, por lo que se comienzan a explorar otras formas de implementarla (Gómez, 2020).

La internacionalización debe priorizarse en la gestión de las universidades públicas y privadas.

\subsection{UNIVERSIDADES LATINOAMERICANAS}

Ante la creciente diversidad y complejidad de las problemáticas que enfrentan las sociedades latinoamericanas, queda claro que los actores del entorno nacional y regional persuaden, directa o indirectamente, para que las universidades y las IES participen más directamente en la solución de los problemas sociales, trascendiendo la noción restringida de colaboración estratégica con el entorno productivo (García \& Lindquist, 2020).

En las tendencias identificadas se devela el interés por el tópico de la investigación en los países de América Latina, en particular Colombia, México, Cuba, Chile, Perú y Venezuela. Las temáticas más abordadas fueron: la formación investigativa, productividad investigativa, representaciones, percepciones y actitudes investigativas y, competencias y habilidades investigativas (López-de Parra, Polanco-Perdomo \& Correa-Cruz, 2017).

Una IES de proximidad, enfocada a la enseñanza en licenciatura, tiene oportunidades de internacionalización distintas a las que tienen los institutos de posgrado y los centros de investigación. Las experiencias de internacionalización que persigan fines de optimización organizacional, de promoción de la equidad social y de innovación productiva deben por lo tanto ser multiplicadas, en torno a líneas prioritarias que otorguen a la internacionalización un carácter de "factibilidad transformativa" (Didou, 2017).

En Latinoamérica, destacan la Universidad de Sao Paulo, Autónoma de México, el Tecnológico de Monterrey y la Universidad de Buenos Aires. Para el caso peruano se revisaron las web de las universidades más destacadas en cuanto a actividad científica y que contaban con información pública en sus páginas web sobre los servicios que prestan. Figuran: la Pontificia Universidad Católica del Perú,... la Universidad Nacional de Ingeniería...y la Universidad Agraria La Molina...(Medina, 2018). 
Las universidades latinoamericanas tienen un compromiso impostergable con la solución de los problemas sociales.

\section{METODOLOGÍA}

La investigación presenta un diseño cualitativo-interpretativo, de tipo documental, el cual precisó el procedimiento de selección y el registro de los datos (Barrero y Rosero, 2018).

En la presente investigación se han seleccionado 52 documentos, realizados en el periodo 2017 2022; incluyendo: artículos científicos, artículos de revisión e información de sitios web de organizaciones reconocidas. Las palabras claves utilizadas en las búsquedas fueron: Internacionalización, Latinoamérica, universidad. Para la selección de los documentos se usaron como criterios: el año de la publicación, la pertenencia con la investigación y ser una fuente confiable. Después de la lectura de cada documento, se procedió a ingresar los datos a la matriz bibliográfica, que sirve para catalogar los documentos de acuerdo con categorías, que se presentan en la tabla 1.

Tabla 1. Matriz bibliográfica

\begin{tabular}{|c|c|c|c|}
\hline Nombre & Tipo & Objetivos & Conclusiones \\
\hline & & & \\
\hline & & & \\
\hline
\end{tabular}

\section{RESULTADOS}

Se presentan los resultados de la internacionalización de las universidades latinoamericanas.

\section{México}

Entre las políticas de internacionalización de la educación superior en México, se encuentra la movilidad estudiantil como un elemento para fortalecer su formación. La internacionalización también se observa en el territorio nacional, con la incorporación de estudiantes, profesionistas y empleadores de otros países que llegan a “competir” por los espacios nacionales (Quiroz, 2014).

La internacionalización, como un eje fundamental para el desarrollo científico, humanístico, académico y cultural de la UNAM, está incorporada en el Plan de Desarrollo Institucional 2015-2019 con 6 líneas de acción y 35 proyectos de desarrollo estratégico. Las políticas internacionales en materia de investigación, educación y colaboración académica y cultural, promueven el desarrollo de la sociedad, siendo éste uno de los principales compromisos de esta casa de estudios (Universidad Nacional Autónoma de México, 2017). 


\section{Guatemala}

La práctica de internacionalización para construir el Modelo Educativo de la USAC, como un elemento para el Desarrollo Curricular de esta institución educativa en Centro América, genera dos aspectos valiosos para las Ciencias de la Educación: la propuesta epistemológica Socio-histórica Cultural en contextos complejos, con el que viven las instituciones de educación superior públicas en América Latina; la incorporación de la dimensión de Gestión de Calidad, como parte de los modelos educativos, debido a la demanda internacional de acreditación de las propuestas curriculares de las instituciones universitarias (Crocker \& Sagastume, 2021).

Con el fin de potenciar los lazos de la Universidad de Santiago con Centroamérica, el Departamento de Relaciones Internacionales se reunió con la delegación de la Universidad de San Carlos proveniente de Guatemala, institución pública con más de 300 años de historia, y que actualmente posee más 150 mil estudiantes (USACH, 2019).

\section{República Dominicana}

Durante el encuentro virtual "La internacionalización de la educación superior”, organizado por el Instituto Tecnológico de Santo Domingo (INTEC), Marmolejos llamó a revisar los planes estratégicos de internacionalización, redefinir una adecuada estructura organizacional, revaluando y revalorar las alianzas estratégicas, adecuar los incentivos y aprovechar sistemas de seguimiento (INTEC, 2020).

La Universidad APEC (UNAPEC), como miembro de la Asociación Dominicana de Rectores de Universidades (ADRU) forma parte de la Red de Internacionalización de Universidades Dominicanas (RIUD-ADRU), que tiene como objetivo general: Fortalecer la internacionalización de la educación superior dominicana, a través de la cooperación e integración, para alcanzar mayor posicionamiento y visibilidad internacional como país (UNAPEC, 2017).

\section{Costa Rica}

La Escuela de Ciencias de la Comunicación Colectiva (ECCC) decidió incorporar la internacionalización como un eje transversal del proceso formativo desde el año 2011, con el fin de incentivar a docentes y estudiantes a participar en proyectos colaborativos e intercambios académicos. Lo anterior ha permitido impulsar una perspectiva global en el desarrollo de las diversas disciplinas de la comunicación, estimular un proceso reflexivo sobre los alcances de la multiculturalidad en el marco de un entorno inclusivo, y adicionalmente, promover el dominio de una segunda lengua (Hütt, 2021).

Para la internacionalización en los programas de posgrado de la Universidad de Costa Rica (UCR), es importante...no solo la movilidad docente hacia el exterior y el intercambio académico con 
universidades del extranjero, sino que, además, se proporcione asesoría y apoyo equitativo y desburocratizado a todos los distintos programas de posgrado para que puedan mejorar las capacidades y las estrategias requeridas para el establecimiento de relaciones internacionales de intercambio y colaboración con universidades, unidades académicas y grupos de investigación de universidades del extranjero (Muñoz, 2018).

\section{Puerto Rico}

La Universidad de Puerto Rico, no está ajena a esta realidad, por lo que ha seguido insertándose en actividades e iniciativas internacionales a través de sus recintos...Sabemos que existen muchos más logros y esfuerzos en el ámbito de la investigación, colaboraciones entre docentes de la UPR y sus homólogos internacionales, publicaciones arbitradas, congresos internacionales y otras actividades académicas y creativas afines al quehacer universitario que no hemos mencionado (Santiago, 2021).

Ante los nuevos retos introducidos por la pandemia, la Universidad del Sagrado Corazón se ha mantenido firme en su misión de aportar a la educación de una comunidad solidaria en la justicia y la paz. Para ayudar a lograrlo, la Oficina de Internacionalización y Relaciones Interuniversitarias (OIRI) crea oportunidades para que los profesores y los estudiantes se conecten con personas y perspectivas de otras culturas (Comunicaciones Integradas, 2021).

\section{Honduras}

Por unanimidad de votos este día el Consejo Universitario aprobó la "Política Institucional de Internacionalización de la Universidad Nacional Autónoma de Honduras (UNAH)”, ,...esta permitió la creación de Vicerrectoría de Relaciones Internacionales, para emprender la tarea fundamental de colocar a la Máxima Casa de Estudios hacia lo internacional, hacia lo global, fortaleciendo todo lo relacionado a la internacionalización de sus procesos académicos, administrativos, de investigación científica y vinculación (Ramirez, 2021).

Roger Enrique Valladares Baker Vicepresidente Ejecutivo de UTH, en representación del presidente de UTH ,Roger D. Valladares firmó hoy en Madrid un convenio de cooperación institucional con la Universidad de Málaga institución de renombre, innovadora que prepara a los profesionales más avanzados que demanda el mercado...Esta semana firmamos convenio también con la Universidad de Córdoba en la ciudad del mismo nombre y también con Loyola en Sevilla (UTH, 2021). 


\section{El Salvador}

La agenda de internacionalización necesita contemplar indicadores que permitan medir en los procesos de internacionalización, resultados que indiquen el nivel de internacionalización de la investigación, por medio de proyectos financiados, proyectos de spin off, asistencia a congresos internacionales que certifique la participación del investigador o grupo investigador, impacto de la investigación aplicadas conjuntas, patentes producto de proyectos conjuntos con instituciones de otros países, publicaciones arbitradas e indexadas; movilidad de investigadores, personal académico, estudiantes, administrativos (Orantes, 2019).

La Universidad Nacional de Agricultura posee convenios académicos internacionales con países de habla hispana, portugués e ingles, con universidades del continente Americano y Europa (UNAG, 2022).

\section{Nicaragua}

En este espacio abordaremos algunos procesos relevantes de la Universidad Nacional Autónoma de Nicaragua, Managua (UNAN-Managua),...La internacionalización de la educación superior en la UNAN-Managua se ha convertido en una plataforma estratégica para concebir y, a la vez, aplicar las funciones de Formación, Investigación y Extensión como procesos transversales en el quehacer Universitario (UNAN, 2021).

Bajo el lema: "Gestionando la presencia y visibilidad de las universidades nacionales en un mundo globalizado", el Consejo Nacional de Universidad, CNU, realizó el I Foro Internacionalización de la Educación Superior en Nicaragua cuya sede fue la UNAN-León...Con este Foro, el Consejo Nacional de Universidades, CNU, pretende crear una sólida base, para socializar, reflexionar y dialogar sobre las diversas prácticas, logros y retos que supone la Internacionalización Universitaria, que permita contribuir a una educación más inclusiva para todas y todos los nicaragüenses (UPOLI, 2021).

\section{Panamá}

La Universidad Tecnológica de Panamá reconoce la internacionalización como la integración de componentes internacionales e interculturales en el proceso de enseñanza-aprendizaje, la investigación, la innovación, la vinculación con la sociedad y con su propia gestión administrativa. Es un factor clave para fortalecer la calidad educativa y mejorar las condiciones de vida de la Sociedad (UTP, 2021).

Con el objeto de suscribir convenios de colaboración y fortalecer la internacionalización, el rector de la Universidad de Panamá (UP), doctor Eduardo Flores Castro, recibió la visita de autoridades de universidades españolas. Firmaron un Convenio Básico de Colaboración que incluye programas para la 
movilidad de investigadores, personal docente y estudiantes, elaboración de proyectos de investigación, organización de actividades docentes y de coloquios internacionales, entre otros (Campos, 2021).

\section{Cuba}

En las últimas décadas, debido al reconocimiento internacional del rigor y nivel del Sistema de Grados Científicos Cubano, se ha ido incrementando de forma sostenida el número de profesionales de otros países que desean realizar sus estudios de doctorado en Cuba y a tal efecto matriculan en instituciones autorizadas cubanas. La Universidad de Cienfuegos, como parte de ese sistema tiene como objetivo, contribuir a la formación de doctores de otras regiones, a partir de la dirección de los procesos de investigación e innovación identificados (Concepción, Rizo \& Capote, 2019).

La internacionalización en el MES ha contribuido al cumplimiento de los objetivos de la educación superior cubana, con un impacto positivo en la formación de estudiantes, en la elevación de la calidad del claustro y en el desarrollo de investigaciones científicas y la innovación en sectores estratégicos para el país. Las acciones se desarrollan bajo el amparo de 2500 convenios interuniversitarios y ministeriales suscritos con instituciones de más de 100 países (MES, 2021).

\section{Colombia}

En la actualidad las políticas públicas educativas consolidan las directrices de la internacionalización y del aseguramiento de la calidad en el contexto de la integración del mercado educativo a nivel global, con tal fuerza política, empuje económico y sustento conceptual, que para un país como Colombia, los desafíos se concentran en los procesos de desarrollo institucional de las instituciones de educación superior, pues, éstas deben adquirir niveles de flexibilidad administrativa, docente e investigativa que se fundamenten más en la sistematización de la información y en la utilización inteligente de ésta información,...( Malagón et al., 2019).

Se presenta una investigación cualitativa descriptiva y documental teniendo como objeto de estudio la Política de Internacionalización (PI) del Instituto Tecnológico Metropolitano (ITM), institución pública adscrita al Municipio de Medellín - Colombia;... Para lograr esto se generan tablas de análisis con los hallazgos encontrados, visualizando un alto grado de coherencia entre los cinco ejes estratégicos de la PI con los ideales propuestos en los documentos de estos organismos internacionales, que incluyen una mirada al entorno global; se concluye que la PI va muy de la mano con los planes de desarrollo nacionales y locales, fortaleciendo características implícitas al desarrollo humano con una perspectiva global (Yepes \& Montes, 2021). 


\section{Venezuela}

Se desarrollaron aspectos de la globalización e internacionalización de la educación superior y de la internacionalización del currículo, como soporte teórico que permitió finalmente generar desde una visión comprehensiva, criterios generales y de nivel microcurricular con el fin de internacionalizar el currículo universitario en la Universidad Yacambú (Mendoza de Gómez \& Villarreal Torres, 2021).

En el caso venezolano, dadas las condiciones presentes en la educación superior del país, el proceso de internacionalización está muy lejos de responder a la internacionalización comprensiva, considerada como la orientación de la misma de cara a los retos de la globalización y de la mercantilización de la educación superior (Parra-Sandoval, 2018).

\section{Ecuador}

En Ecuador, la Universidad Técnica de Manabí la utiliza para mejorar su desempeño. El objetivo perseguido por este estudio fue evaluar la efectividad de la estrategia de internacionalización en el progreso de la formación postgraduada y la producción científica en esta universidad y conocer su alineación a las necesidades territoriales y del país...Se comprobó que un porciento alto de publicaciones en bases de datos regionales es realizado por doctorandos y maestrantes, y que existe relación entre sus temas de investigación y problemas del territorio, a diferencia de Scopus (López, Pelegrín \& Ruiz, 2021).

En el Ecuador según el INEC se registra un incremento constante de personas que viajan fuera del país por motivos de estudios. Un comparativo del año 2000 al año 2015 denota un interés del ecuatoriano por estudiar en el exterior, incrementando de 669 a 13.987 personas (Ayala Bolaños \& Valencia, 2018).

\section{Perú}

Se reflexionó la necesidad de reconocer en los cambios una oportunidad de mejora que dialogue con las políticas mundiales y nacionales de la internacionalización en los programas de posgrado que fomente la creación de redes de conocimiento entre personas que no se conocen mediante un interesante concepto: el proceso de intercambio (Briñez y Guerrero, 2021).

Desde la UPC nos sumamos al foro "Desarrollo de la Internacionalización en las universidades peruanas: estructuras, políticas y acciones”, organizado por la Red Peruana de Universidades Nacionales para la Internacionalización - RUNAi, presidida por la Universidad Nacional Mayor de San Marcos (UNMSM); en alianza con REDIPerú; la Universidad de Ingeniería y Tecnología (UTEC); y la Universidad Peruana de Ciencias Aplicadas (UPC); con el auspicio de la Embajada de los Estados Unidos de América en el Perú y el Ministerio de Educación. Este Foro busca contribuir con el sistema educativo nacional y su proceso de internacionalización,...( Noticias UPC, 2021). 


\section{Brasil}

De acuerdo con los parámetros de la Universidad del Oeste de Santa Catarina (Unoesc), para colaborar con el desarrollo de la internacionalización, la institución busca implementar proyectos que permitan su avance. Uno de ellos es el Proyecto de Movilidad Académica creado en 2010 como una de las primeras acciones de internacionalización de la Universidad, que, con este Programa y la institución de un coordinador específico para planificar y ejecutar acciones estratégicas, asumió el compromiso con el Desarrollo y la formación humana de estudiantes y profesores (Freitas, Pinto de Almeida, Palmeira Rodrigues \& Pezavento, 2021).

Antonio José de Almeida Meirelles, rector de la Universidade Estadual de Campinas (UNICAMP) y vicepresidente de la Región Brasil de la UDUAL, aseguró que las universidades fueron capaces de enfrentar positivamente los retos de la pandemia y de ayudar a la sociedad a enfrentar los desafíos políticos que gestionan los destinos de la ciencia y la tecnología. Destacó que la UDUAL es una institución que mantiene unidas a las universidades de América Latina y el Caribe (ALC) y subrayó que se debe valorar a la ciencia y a la educación como grandes instrumentos de transformación de nuestras realidades (UDUAL, 2021).

\section{Bolivia}

Bolivia tuvo su contraparte, en 1931, se decreta la autonomía universitaria por el gobierno del Gral. Blanco Galindo, después, si bien la universidad boliviana gozaba de su autonomía, poco o casi nada se hizo en la modernización de sus programas académicos, ni estructurales y con una actitud incompresible se divorció con las aspiraciones del pueblo, solo en la época de los años 70s y 80s la universidad boliviana tuvo una activa participación en la vida política del país teniendo una actitud más contestataria y revolucionaria (Campohermoso-Rodríguez et al., 2019).

Se describe el desarrollo en Univalle del Programa de Movilidad Académica Regional para Carreras de Grado Acreditadas por el Sistema de Acreditación Regional del Mercado Común del Sur MARCA- MERCOSUR... Se desarrolla la experiencia institucional como Coordinadores Generales de las redes de Medicina, Odontología y Electrónica. Lo propio para este último periodo 2018-2019, en el cual se pudo compartir la experiencia aprendida a las universidades bolivianas públicas y privadas acerca de cómo llegar a ser parte de las distintas redes de intercambio (Vélez, 2019).

\section{Uruguay}

Desde el Vicerrectorado de Relaciones Internacionales de la Universidad de Concepción del Uruguay nos hemos postulado a la convocatoria promovida por el Programa de Internacionalización de 
la Educación Superior y Cooperación Internacional (PIESCI), denominada "Fortalecimiento de los procesos de internacionalización de las universidades 2021 ”... Este proyecto tiene por finalidad, poder consolidar un camino de internacionalización "en casa", promoviendo y fortaleciendo los lazos con nuestros socios extranjeros, a partir de acciones conjuntas que signifiquen un salto de calidad en nuestras aulas y espacios de gestión e investigación de la Universidad (UCU, 2021).

Universidad de Concepción del Uruguay, la UCU se ha afianzado en la región contando actualmente con seis facultades, cuatro centros regionales y una extensión áulica en constante expansión. Asimismo, ha iniciado un proceso de inserción en el ámbito internacional, tanto en el plano cultural como en el académico, mediante emprendimientos conjuntos con diversas universidades del exterior, especialmente de Europa y el MERCOSUR (Franzante, Carbone \& Perdomo, 2021).

\section{Paraguay}

En este sentido, el ámbito del Sector Educativo del Mercosur, ha brindado respuestas acordes a la realidad del sistema de Educación Superior de Paraguay, en la medida en que se presenta como instancia legitimadora de un sistema de Educación Superior cuestionado por su misma trayectoria sociohistórica, al mismo tiempo que ofrece alternativas a las dinámicas de internacionalización de las universidades públicas, a partir de favorecer la generación de circuitos regionales o redes de producción y difusión de conocimiento (Oregioni y Avondet, 2021).

La práctica en las universidades paraguayas indica que, con miras a la internacionalización curricular resulta necesaria la incorporación de estándares internacionales en los contenidos de asignaturas, así como en los métodos docentes más eficientes, la revisión y análisis de las tendencias internacionales, tratando de adoptarlas y esencialmente adecuarlas dentro de las posibilidades y características propias, sin descuidar por tanto la realidad local, con miras a lograr en la comunidad educativa en general y los estudiantes en particular (Alvarenga, 2019).

\section{Argentina}

Si bien la apertura unilateral recortó espacios de autonomía universitaria; por la otra, generó incentivos para que las IES promovieran una mayor articulación con el sector productivo y con la esfera internacional. Estas oportunidades de internacionalización (externa e interna) de las IES se ampliaron aún más con el reforzamiento del Mercosur Educativo que abrió nuevas ventanas y oportunidades para la cooperación entre los países y la competencia entre las IES (Botto, 2017).

La internacionalización de la Educación Superior es un eje fundamental, dentro de las estrategias definidas por las universidades de todo el mundo, como uno de los mecanismos de respuesta al proceso 
de globalización. La UNA (Universidad Nacional de Asunción) busca promover la internacionalización a través de las acciones y en contacto directo con el Rectorado y las Unidades Académicas de la Universidad. La Universidad cuenta con una política de internacionalización clara a través de la apertura a las universidades del mundo y especialmente a las acciones de movilidades de estudiantes (grado y postgrado), docentes, investigadores y gestores administrativos (UNA, 2021).

\section{Chile}

Analiza los procesos de internacionalización de la Universidad Adventista de Chile, con énfasis en período de pandemia...Sobre el 50\% de las instancias de internacionalización se dan por convenios, evidenciado que hay un umbral de relaciones institucionales que pueden consolidarse a partir de convenios entre universidades e instituciones (Gutiérrez, 2021).

Ennio Vivaldi aseguró que la pandemia deja claro que la internacionalización de las universidades ya no es solo una opción, sino que es una necesidad para poder solucionar los problemas globalmente. "Para la fluidez de las relaciones internacionales se necesita que fomentemos que los estudiantes, sobretodo de pre y postgrado, puedan tener oportunidades de intercambio, puedan tener a una microescala un estudio de comparación internacional sobre cómo son sus propias costumbres respecto a las de otras países", señaló el Rector de la Universidad de Chile (USACH, 2021).

Las variables clave a considerar por las instituciones de educación superior latinoamericanas con el fin de lograr una efectiva influencia de la internacionalización en los procesos universitarios, tales como: globalización de la educación superior, cambios estructurales en la universidad e internacionalización como proceso estratégico institucional (Llano et al., 2021).

Así, en nuestros países latinoamericanos aparecen experiencias que promueven formas solidarias de internacionalización, con una clara vocación contrahegemónica, y que constituyen una apuesta a cimentar el sistema de educación superior en principios de solidaridad, igualdad y justicia social (Prados, 2018).

Los procesos de internacionalización en las universidades latinoamericanas, cobran mayor importancia durante la pandemia, y también, demuestran un esfuerzo sostenido de estas instituciones.

La internacionalización debe priorizarse en la gestión de las universidades públicas y privadas.

\section{CONCLUSIONES}

Dentro de las políticas de internacionalización de las universidades latinoamericanas, tenemos la movilidad estudiantil, investigación, educación y colaboración académica y cultural, la cooperación e integración, participar en proyectos colaborativos e intercambios académicos con universidades del 
extranjero, firma de convenios académicos internacionales, contribuir a una educación más inclusiva, integración de componentes internacionales e interculturales, coloquios internacionales, internacionalizar el currículo universitario, generación de circuitos regionales o redes de producción y difusión de conocimiento.

Es importante y urgente, que las universidades latinoamericanas, trabajen de manera coordinada y conjunta para lograr mejores resultados en sus procesos de internacionalización. 


\section{REFERENCIAS}

Ayala Bolaños, M., \& Valencia Cruzaty, L. (2018). La Internacionalización, una perspectiva para mejorar la calidad de la educación superior en el Ecuador. REVISTA CIENCIAS PEDAGÓGICAS E INNOVACIÓN, 6(2), 61-69. https://doi.org/10.26423/rcpi.v6i2.254

Alvarenga, L. (2019). El desafío de la internacionalización curricular en la facultad de derecho de la Universidad Nacional del Este, Paraguay. Congresso de Internacionalização da Educação Superior. Recuperado de http://dspace.unila.edu.br/123456789/5717

Barrero, A. y Rosero, A. (2018). Estado del Arte sobre Concepciones de la Diversidad en el Contexto Escolar Infantil. Revista Latinoamericana de Educación Inclusiva, 2018, 12(1), 39-55 https://doi.org/10.4067/S071873782018000100004

Botto, Mercedes. (2017). La internacionalización de la educación superior en la Argentina (1995-2015): ¿Convergencia global o peculiaridades nacionales?. Actualidades Investigativas en Educación, 17(3), 795 818. https://dx.doi.org/10.15517/aie.v17i3.29930

Briñez, K. y Guerrero, R. (2021). Experiencia de una estrategia de internacionalización en programas de posgrado de Enfermería: Intercambio cultural y de aprendizaje. https://doi.org/10.35383/cietna.v8i2.668

Campos, I. (2021). La Universidad de Panamá firmó convenio con universidades españolas. Recuperado de https://upinforma.com/nuevo/info.php?cat=noticias\&id=6263

Campohermoso-Rodríguez O F, Soliz-S. R E, Campohermoso-R. O, Flores-H. R I, Huallpara-S. V. (2019). A más de cien años de la autonomía universitaria en Latinoamérica. Cuad. - Hosp. Clín. vol.60 no.1. Recuperado de http://www.scielo.org.bo/scielo.php?script=sci_arttext\&pid=S1652-67762019000100012

Comunicaciones Integradas (2021). Sin pausa la internacionalización en Sagrado. Recuperado de https://insagrado.sagrado.edu/sin-pausa-la-internacionalizacion-en-sagrado/

Comas Rodríguez, Oscar Jorge. (2019). La internacionalización de la educación superior. Revista de la educación superior, 48(192), 165-168. Epub 30 de enero de 2020. Recuperado de http://www.scielo.org.mx/scielo.php?script=sci_arttext\&pid=S0185-27602019000400165\&lng=es\&tlng=es. 
Concepción Morales, E. R., Rizo Rabelo, N., \& Capote León, G. E. (2019). La internacionalización en la Universidad de Cienfuegos: una vía para el fortalecimiento de la gestión académica e investigativa. Universidad y Sociedad, 11(1), 67-74. Recuperado de http://rus.ucf.edu.cu/index.php/rus

Crocker Sagastume, R. C., \& Sagastume Lizama, R. del C. (2021). Construcción del modelo educativo de una universidad guatemalteca con criterios de internacionalización curricular. Journal of the Academy, (5), 156172. https://doi.org/10.47058/joa5.10

Didou Aupetit, Sylvie (2017). Vincular la internacionalización con las prioridades de desarrollo de las instituciones de Educación Superior: una urgencia inaplazable. Educação, 40(3),324-332. [fecha de Consulta 10 de Enero de 2022]. ISSN: 0101-465X. Disponible en: https://www.redalyc.org/articulo.oa?id=84854915007

Franzante, B., Carbone, G. A., \& Perdomo Vázquez, J. M. (2021). La investigación en línea camino para la internacionalización. Revista Educación Superior Y Sociedad (ESS), 33(2), 824-849. https://doi.org/10.54674/ess.v33i2.368

Freitas, S. T., Pinto de Almeida, M. de L., Palmeira Rodrigues, D., \& Pezavento, K. (2021). Internacionalización de la educación superior en el oeste de Santa Catarina: la experiencia de una universidad comunitaria. Revista Educación Superior Y Sociedad (ESS), 33(2), 850-874. https://doi.org/10.54674/ess.v33i2.382

García Galván, Rodolfo, \& Lindquist Sánchez, Ricardo. (2020). Hacia una agenda social de las universidades latinoamericanas del siglo XXI: una perspectiva teórica-epistémica y política. Revista de la educación superior, 49(194), 89-113. Epub 27 de noviembre de 2020.https://doi.org/10.36857/resu.2020.194.1126

Gómez, A. (2020). ¿Qué sucederá con la internacionalización de la educación superior y la movilidad estudiantil después del COVID-19?. Revista Digital de Investigación en Docencia Universitaria. 14(2), e1348. https://doi.org/10.19083/ridu.2020.1348

Gutiérrez, N. et al. (2021). Internacionalización universitaria en contexto de pandemia. Un caso en Chile. Podium. DOI: 10.31095/podium.2021.40.7

Hütt, H. (2021). Internacionalización. ¿Qué es la Estrategia de Internacionalización de la ECCC?. Recuperado de https://eccc.ucr.ac.cr/internacionalizacion/ 
INTEC (2020). Internacionalización de la educación superior más que una estructura es una cultura. Recuperado de https://www.intec.edu.do/prensa/notas-de-prensa/item/internacionalizacion-de-la-educacionsuperior-mas-que-una-estructura-es-una-cultura

López Montero, Rosberly (2018). Propuesta de internacionalización desde las estrategias didácticas universitarias. ALTERIDAD. Revista de Educación, 13(2), 239-250. https://doi.org/10.17163/alt.v13n2.2018.07

López-de Parra, L., Polanco-Perdomo, V., \& Correa-Cruz, L. (2017). Mirada de las investigaciones sobre formación investigativa en la universidad latinoamericana: estado del arte (2010-2017). Rev.investig.desarro.innov, 8(1), 77-95. doi: 10.19053/20278306.v8.n1.2017.7371

López, L., Pelegrín, N. \& Ruiz, M. (2021). Relación entre formación de postgrado internacional e investigación en universidades de Ecuador. Ingeniería Industrial: Vol. 42 Núm. 1 (2021). Recuperado de https://rii.cujae.edu.cu/index.php/revistaind/article/view/1077

Llano Zhinin, Gladys Verónica, Carlozama Puruncajas, Jorge Fernando, Tipán Cañaveral, Daniel Fernando, \& Tipán Cañaveral, Gustavo Bernardo (2021). La internacionalización en los procesos universitarios: un análisis estructural para universidades de Latinoamérica. Mendive. Revista de Educación, 19(1), 6-15. Epub 02 de marzo de 2021. Recuperado de http://scielo.sld.cu/scielo.php?script=sci_arttext\&pid=S1815$76962021000100006 \& \operatorname{lng}=\mathrm{es} \& \operatorname{tlng}=\mathrm{es}$.

Macazana Fernández, Dante Manuel, Romero Diaz, Alejandra Dulvina, Vargas Quispe, Guillermo, Sito Justiniano, Luz Marina, \& Salamanca Chura, Edith Cristina. (2021). Procedimiento para la gestión de la internacionalización de la educación superior. Dilemas contemporáneos: educación, política y valores, 8(spe2), 00003. Epub 21 de abril de 2021.https://doi.org/10.46377/dilemas.v8i.2585

Malagón Plata, Luis Alberto, Rodríguez Rodríguez, Luz Helena, \& Machado Vega, Diego Fernando. (2019). Políticas Públicas Educativas y aseguramiento de la calidad en la Educación Superior. Revista Historia de la Educación Latinoamericana, 21(32), 273-290. https://doi.org/10.19053/01227238.4999

Medina Coronado, Daniela. (2018). El rol de las universidades peruanas frente a la investigación y el desarrollo tecnológico. Propósitos y Representaciones, 6(2), 703-720. https://dx.doi.org/10.20511/pyr2018.v6n2.244 
Mendoza de Gómez, B., \& Villarreal Torres, M. J. (2021). internacionalización del curriculo para la formaciòn de competencias globales. Red De Investigación Educativa, 13(2), 65 - 78. Recuperado a partir de https://revistas.uclave.org/index.php/redine/article/view/3309

MES (2021). Internacionalización. Recuperado de https://www.mes.gob.cu/internacionalizacion-0

Muñoz Varela, Luis. (2018). Capacidades y estrategias de internacionalización del posgrado en la Universidad de Costa Rica. Actualidades Investigativas en Educación, 18(1), 99-132. https://dx.doi.org/10.15517/aie.v18i1.31796

Noticias UPC (2021). Universidades nacionales e internacionales se reúnen para potenciar la transformación de la internacionalización en la educación superior en el Perú. Recuperado de https://noticias.upc.edu.pe/2021/09/10/universidades-nacionales-e-internacionales-se-reunen-para-potenciarla-transformacion-de-la-internacionalizacion-en-la-educacion-superior-en-el-peru/

Orantes, B. R. (2019). Retos de la internacionalización universitaria para América Latina en el marco de la cooperación Sur-Sur. Recuperado de https://www.rlcu.org.ar/recursos/Articulo_Internacionalizacion.pdf

Oregioni, M. S. (2017). La internacionalización universitaria desde una perspectiva situada: tensiones y desafíos para la región Latinoamericana. Revista Internacional De Educação Superior, 3(1), 114-133. https://doi.org/10.22348/riesup.v3i1.7667

Oregioni, S. y Avondet, L. (2021). Estímulos a la internacionalización y criterios de calidad en las universidades de Paraguay.Integración y Conocimiento,10 (2), 264-283

Parra-Sandoval, M. (2018). Gobernanza y Gestión Universitaria en Latinoamérica. Revista Venezolana de Gerencia, vol. Esp, núm. 1. Recuperado de https://www.redalyc.org/articulo.oa?id=29062781003

Prados, María Luz (2018). La internacionalización de la universidad: debates globales, acciones regionales. Revista Brasileira de Educação [online]. Disponible en: 〈https://doi.org/10.1590/S1413-24782018230100>.

Quiroz, E. (2014). Internacionalización e interculturalidad. Reencuentro. Análisis De Problemas Universitarios, (67), 59-64. Recuperado de https://reencuentro.xoc.uam.mx/index.php/reencuentro/article/view/838 
Ramirez, J. (2021). Consejo Universitario aprobó la Política Institucional de Internacionalización de la UNAH. Recuperado de https://presencia.unah.edu.hn/noticias/consejo-universitario-aprobo-la-politica-institucionalde-internacionalizacion-de-la-unah/

Santiago, F. (2021). Internacionalización de la Universidad de Puerto Rico. Recuperado de https://www.upr.edu/internacionalizacion-de-la-universidad-de-puerto-rico/

UCU (2021). Fortalecimiento de los procesos de internacionalización de las Universidades (2021). Recuperado de https://ucu.edu.ar/fortalecimiento-de-los-procesos-de-internacionalizacion-de-las-universidades-2021/

UDUAL (2021). La UDUAL llevó a cabo su VI Reunión Regional Brasil-Cono Sur. Recuperado de https://www.udual.org/principal/2021/11/11/la-udual-llevo-a-cabo-su-vi-reunion-regional-brasil-cono-sur/

UNA (2021). Internacionalización. Recuperado de https://www.una.py/internacional

UNAG (2022). Convenios. Recuperado de https://internacionalizacion.unag.edu.hn/convenios/

UNAN (2021). Internacionalización transversal en la UNAN-Managua: Un proceso sin precedentes y permanente. Recuperado de https://www.unan.edu.ni/index.php/articulos-entrevistasreportajes/internacionalizacion-transversal-en-la-unan-managua-un-proceso-sin-precedentes-ypermanente.odp

UNAPEC (2017). UNAPEC forma parte de la Red de Internacionalización de Universidades Dominicanas (RIUD-ADRU). Recuperado de https://unapec.edu.do/noticias-historico/unapec-forma-parte-de-la-red-deinternacionalizaci\%C3\%B3n-de-universidades-dominicanas-riud-adru/

Universidad Nacional Autónoma de México (2017). Políticas de internacionalización. Recuperado de https://www.unaminternacional.unam.mx/es/politicas

UPOLI (2021). UPOLI en I Foro Internacionalización de la Educación Superior en Nicaragua. Recuperado de https://www.upoli.edu.ni/noticias/ver/1012-upoli-en-i-foro-internacionalizacion-de-la-educacion-superioren-nicaragua 
USACH (2021). Nuevos paradigmas en la internacionalización universitaria post-pandemia. Recuperado de https://www.drii.usach.cl/es/noticia/nuevos-paradigmas-en-la-internacionalizaci\%C3\%B3n-universitariapost-pandemia

USACH (2019). Representantes de la Universidad de San Carlos de Guatemala visitan la Usach para establecer planes de cooperación a future. Recuperado de https://www.drii.usach.cl/es/noticia/representantes-de-launiversidad-de-san-carlos-de-guatemala-visitan-la-usach-para-establecer

UTH (2021). UTH suma tres instituciones españolas a su creciente proceso de internacionalización. Recuperado de https://www.uth.hn/uth-suma-tres-instituciones-espanolas-a-su-creciente-proceso-deinternacionalizacion/

UTP (2021). Internacionalización. Recuperado de https://utp.ac.pa/internacionalizacion

Vélez Ocampo V., M. (2019). El impacto de la experiencia MARCA en Bolivia: La Universidad Privada del Valle. Integración Y Conocimiento, 8(1), 33-47. Recuperado de https://revistas.unc.edu.ar/index.php/integracionyconocimiento/article/view/24660

Yepes Zuluaga, S. M., \& Montes Granada, W. F. (2021). Análisis estratégico de la política de internacionalización en una institución de educación superior en Colombia. Academia Y Virtualidad, 14(2), 109-124. https://doi.org/10.18359/ravi.5456 